\title{
The control system design of the wheel-legged robot
}

\author{
Zhang $\mathrm{Fu}^{1}$, Fu Sanling ${ }^{2}$, Liu Yong ${ }^{3}$, Xue Kunpeng ${ }^{1}$, Zhang Yakun ${ }^{1}$ \\ ${ }^{1}$ (College of Vehicle \&Motive Power Engineering, Henan University of Science and Technology, Luoyang, P R \\ China) \\ ${ }^{2}$ (College of Physics and Engineering, Henan University of Science and Technology, Luoyang, P R China) \\ ${ }^{3}$ (Great Wall Motor Company Limited (GWM), Baoding, P R China)
}

\begin{abstract}
The wheel-legged robot had deep integration of their respective advantages between the wheeled robot and the legged robot. The control system of the wheel-legged robot is the core contents of research, so the control system was designed and researched in the paper. AT89C52 single chip microcomputer was used as the control center in the control system of the wheel-legged robot. The control system mainly included the remotecontrol unit, the order processing system, the actuator control system. The order processing system will send orders for the actuator control system when the system had received the signal of the remote control system, and so that the robot can accomplish the scheduled action. The experimental results showed that the maximum error of the PWM signal controlling actuator is less than $l^{\circ}$ under the crystal oscillator frequency for $24 M$ conditions.
\end{abstract}

Keywords — wheel-legged robot, control system, software design, hardware design

\section{INTRODUCTION}

Recently, with the progress of grounded theory and unit technology, intelligent robot has been applied widely in most social fields. The robot has generally divided into two categories: the legged robot and the wheeled robot depending on ground contact parts, and two robots have their own advantages and disadvantages. The advantages of the legged robot included walking the flat ground or the rugged ground, spanning the ravine, ascending and descending the stairs, non-sliding of complete and unidirectional movement, and so on, which was used as the most fields of the society, but the robot has shortcomings that is difficult to achieve the stable gait planning and stable equilibrium control, in addition, the motion velocity and energy utilization efficiency of the legged robot are low owing to the overall weight and the complex structure ${ }^{[1-2]}$. The wheeled robot as another robot has many advantages included the automatic simple operation, the stable movement, the more motion velocity and energy utilization efficiency, so which is especially suitable for motion in the flat ground, but compared with the legged robot, the disadvantages included the poor adaptability of the terrain ${ }^{[3-6]}$.

With the continuous development of society, the convenient, rapid wheel-leg transform robot was designed in order to solve complex road walking problems, and the robot had deep integration of their respective advantages of the wheeled robot and the legged robot, at the same time, also made up the shortcomings between the wheeled robot and the legged robot, so the wheel-legged robot has a great significance. The control system of the robot is the core contents of research, so the control system was designed and researched in the paper.

\section{THE WORKING PRINCIPLE OF THE CONTROL SYSTEM}

AT89C52 single chip microcomputer was used as the control center in the control system of the wheellegged robot. The control system mainly included the remote-control unit, the order processing system, the actuator control system. The order processing system will send orders for the actuator control system when the system had received the signal of the remote control system, and so that the robot can accomplish the scheduled action.

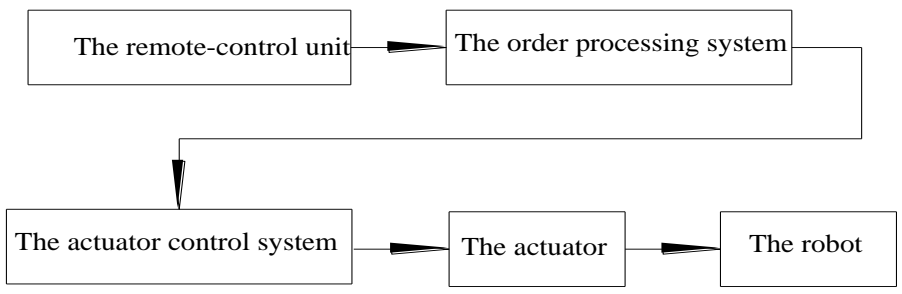

Fig. 1 the control flow diagram 


\section{1 the remote control system}

\section{THE CIRCUIT DESIGN}

The remote control system is composed of 52 single chip microcomputer, the keys and the wireless communication module. NRF24L01 was used as the wireless module, which works on the frequency range from $2.4 \mathrm{GHz}$ to $2.5 \mathrm{GHz}$. The wireless function module included the built-in frequency synthesizer, the power amplifier, the crystal oscillator, the modulator, and so on. The embedded baseband protocol engine (Enhanced ShockBurst) is based on packet communication and supports various modes from manual operation to advanced autonomous protocol operation. Internal FIFOs ensure a smooth data flow between the radio front end and the system's MCU. Enhanced ShockBurst reduces system cost by handling all the high-speed link layer operations and combined with enhanced ShockBurst technology, the output power and communication channel can be through the procedures configuration. The air data rate supported by the NRF24L01 is configurable to $2 \mathrm{Mbps}$. The high air data rates combined with two power saving modes makes the NRF24L01 very suitable for ultra low power designs. Internal voltage regulators ensure a high Power Supply Rejection Ratio and a wide power supply range. NRF24L01 electrical characteristics were showed as Tab. 1. NRF24L01 pin assignment, the circuit diagram of the remote control system and the circuit diagram of the receiving system was showed as Fig.2, Fig. 3 and Fig. 4 respectively.

Tab. 1 NRF24L01 electrical characteristics

\begin{tabular}{lc}
\hline \multicolumn{1}{c}{ parameters } & contents \\
\hline Minimum power supply voltage & $1.9 \mathrm{~V}$ \\
maximum Data Transfer Rate & $2000 \mathrm{kbps}$ \\
current consumption at the Emission mode $(0 \mathrm{dBm})$ & $11.3 \mathrm{~mA}$ \\
current consumption at the receiving mode $(2000 \mathrm{kbps})$ & $12.3 \mathrm{~mA}$ \\
Temperature range & $-40 \sim+85^{\circ} \mathrm{C}$ \\
The sensitivity of 1000 kbps data transfer rate & $-85 \mathrm{~dB}$ \\
current consumption at power down mode & $900 \mathrm{nA}$ \\
\hline
\end{tabular}

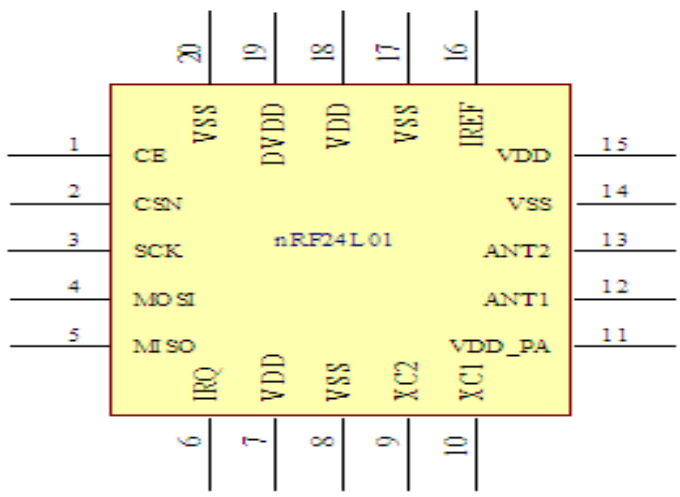

Fig. 2 NRF24L01 pin assignment

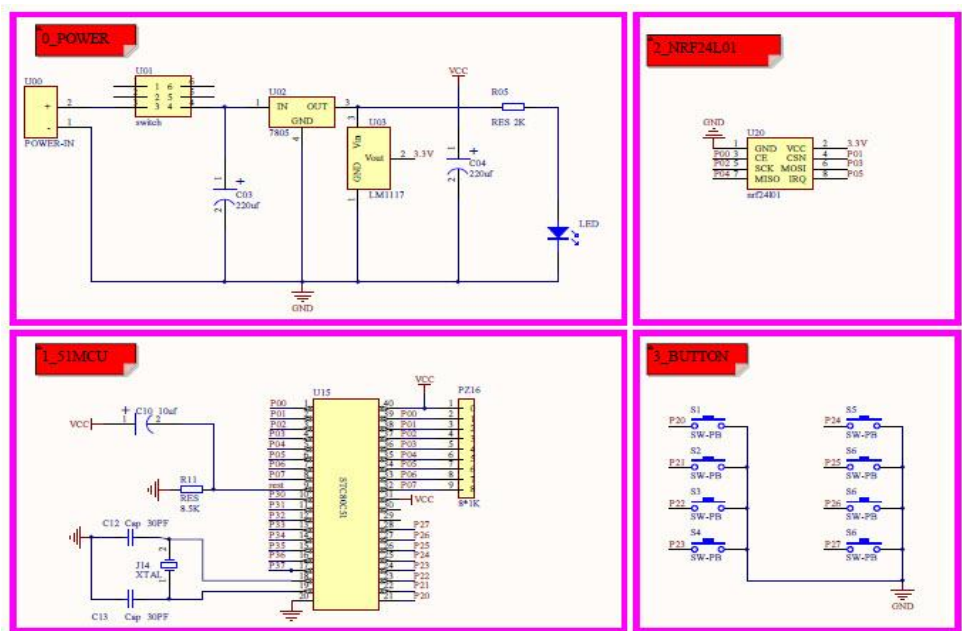

Fig. 3 the circuit diagram of the remote control system 


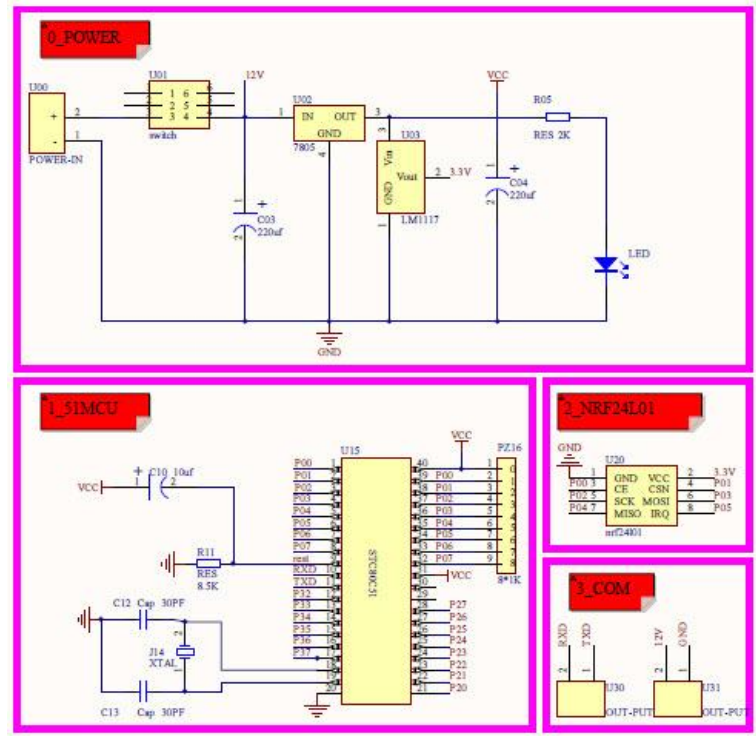

Fig. 4 the circuit diagram of the receiving system

\section{2 the order processing system and the actuator control system}

The order processing system and the actuator control system is mainly composed of 52 single chip microcomputer, the system receives the command from the remote control through the wireless communication module, and the analysis results of the commands was loaded the corresponding subprogram, and the instruction of the robot execution was concluded by the subprogram which will transfer to the actuator control system through the serial communication mode. The actuator control system had received the instruction from the order processing system which will transfer the signal of duty cycle for the actuator movement, so the robot can accomplish the action. The flow chart between the order processing system and the actuator control system was showed as Fig. 5.

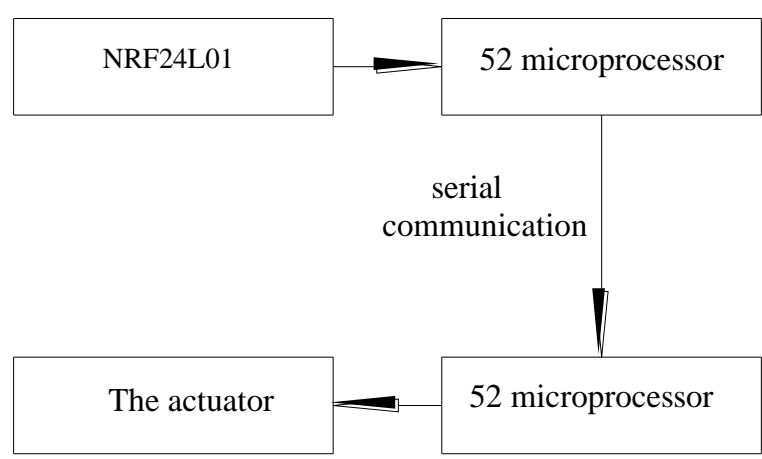

Fig. 5 the flow chart between the order processing system and the actuator control system

\section{THE SOFTWARE DESIGN}

\section{1 the software design of the remote control and the wireless communication module}

The wireless transmitting software included the initialization operation, the working mode setting, and so on. The initialization operation has two parts included the SCM (single computer microprocessor) I/O setting and SPI related register which can communicate with NRF24L01, and the RF chip was set by the SPI bus which can enter the correct work mode. Firstly, NRF24L01 configuration mode was set as transmitting mode when the remote control system will transmit the data, then the data destination address TX-ADDR and data TX-PLD of the awaiting emission data was loaded in NRF24L01 buffer, and delay after the launch data. The flow chart of the wireless transmitting software was showed as Fig. 6 . 


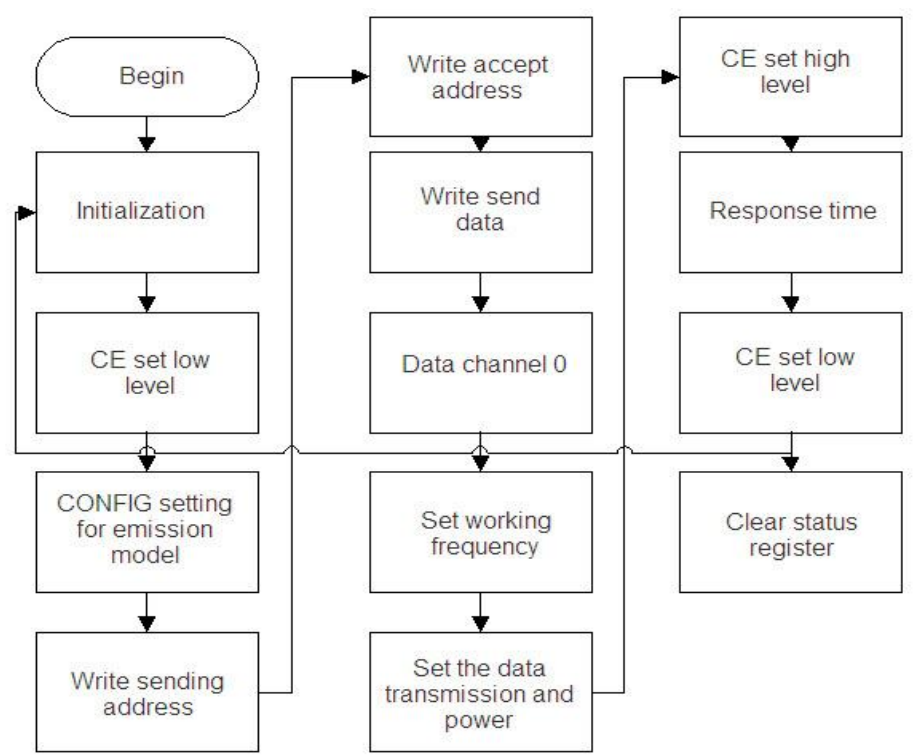

Fig. 6 the flow chart of the wireless transmitting software

NRF24L01 configuration mode was set as receiving mode when the system will receive the data, and then delay in receiving state waiting for the arrival of the data. The data packet will be stored in the stack when the system detected effective address and CRC, and the interrupt flag RX-DR of state register will set high level, and produce interrupt make IRQ pins into low level to inform single chip microcomputer to receive the data. The flow chart of the wireless receiving software was showed as Fig. 7.

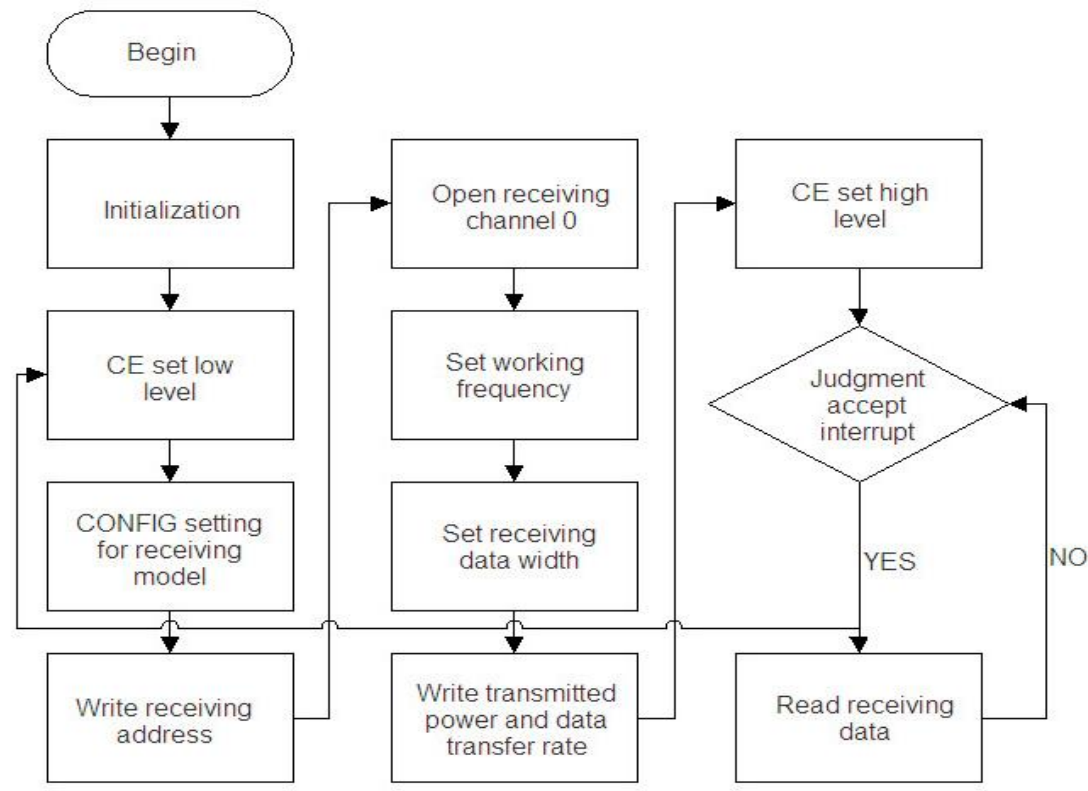

Fig. 7 the flow chart of the wireless receiving software

\section{2 the software design of the order processing system and the actuator control system}

The subprogram will execute when the SCM received from the remote control command, and the execution position and the execution speed of instructions of various actuators was concluded by the subprogram, then transfer to 52 microprocessor of the actuator control system through the subprogram of the serial communication.

The duty ratio signal of each actuator will concluded based on the instructions of the microprocessor of the actuator control system, and the signal will through the pin sent to each actuator, and so that the wheellegged robot can accomplish the scheduled action.

Standard actuator has three lines included the power line, the ground line and the signal control line. The control signal line need to enter PWM signal in order to use the duty ratio controlling the position of the actuator. The PWM signal of the actuator is mostly a period of $20 \mathrm{~ms}$ pulse width modulation signal, the Output 
Angle of the actuator will range from $0^{\circ}$ to $180^{\circ}$ when the signal line of the actuator was transfer PWM signal of the pulse width between $0.5 \mathrm{~ms}$ and $2.5 \mathrm{~ms}$. The relation between output Angle and pulse width can be simple expressed as:

$$
A=90(t-0.5)
$$

In the formulas, where, $A$ is the output angle of the actuator; $t$ is the pulse width, $0.5 \leq t \leq 25$; and we can see that there is a liner relation between the output angle of the actuator and the pulse width.

At the same time, the time-sharing multiplexing algorithm was adopted in order to accurately control multiple actuators position and speed in the microprocessor of the actuator control system. The 20ms period of one PWM signal will divide into eight time parts, and each time parts will parallelly produce three-channel PWM signals, so twenty-four-channel PWM signals will produce. The research on the wheel-legged robot need twenty actuators, the PWM signals can meet the requirements. The experimental results showed that the maximum error of the PWM signal controlling actuator is less than $1^{\circ}$ under the crystal oscillator frequency for $24 \mathrm{M}$ conditions. The emission flow chart of the order processing system software and the receiving flow chart of the order processing system software were showed as Fig. 8 and Fig. 9 respectively.

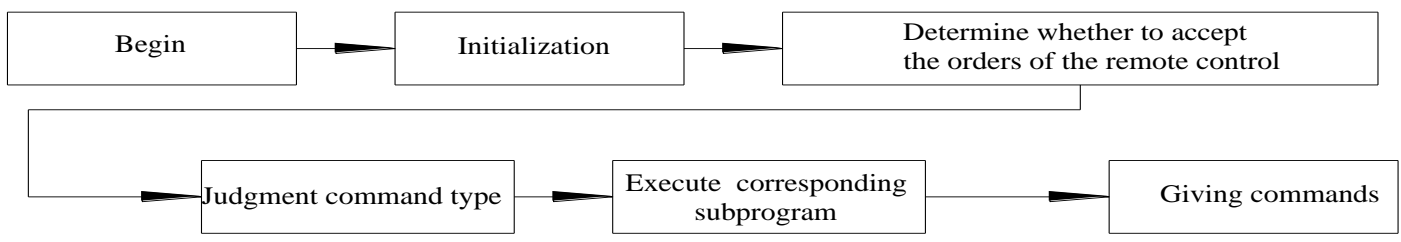

Fig. 8 the emission flow chart of the order processing system software

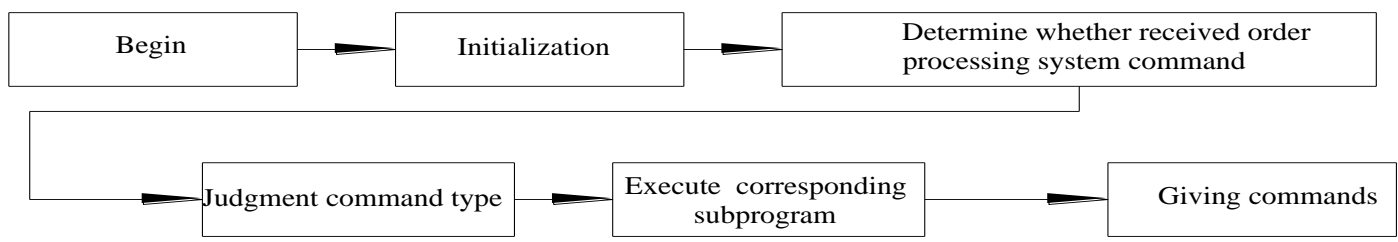

Fig. 9 the receiving flow chart of the order processing system software

\section{CONCLUSION}

The control system of the wheel-legged robot was designed, and the research can realize the wireless remote control, the wheeled motion control, the legged motion control and the switch between wheeled condition and legged condition of the wheel-legged robot. Two-way communication was realized by the NRF24L01 wireless communication module, and the order processing system and the actuator control system was realized by two 52 microprocessor respectively.

\section{ACKNOWLEDGEMENTS}

This work was supported by Scientific Research Foundation for Ph. Doctor and Student Research Training Project (SRTP) of Henan University of Science and Technology.

\section{REFERENCES}

[1] Zhiguo Shi, Qiao Zhang and Jun Tu, et al, Vision Stability of Four-legged Robot Based on the Feedback of Motion Parameters, Procedia Engineering, 29,2012, 3250-3255.

[2] Umberto Scarfogliero, Cesare Stefanini and Paolo Dario, The use of compliant joints and elastic energy storage in bio-inspired legged robots, Mechanism and Machine Theory, 44(3) 2009, 580-590.

[3] M. Haddad, T. Chettibi, S. Hanchi, et al. A random-profile approach for trajectory planning of wheeled mobile robots. European Journal of Mechanics-A/Solids, 26(3), 2007, 519-540.

[4] Zenon Hendzel and Marcin Szuster. Discrete neural dynamic programming in wheeled mobile robot control. Communications in Nonlinear Science and Numerical Simulation, 16(5), 2011, 2355-2362.

[5] Gregor Klančar, Drago Matko and Sašo Blažič. A control strategy for platoons of differential drive wheeled mobile robot. Robotics and Autonomous Systems, 59(2), 2011, 57-64.

[6] Alexey S Matveev and Hamid Teimoori, Andrey V Savkin. Range-only measurements based target following for wheeled mobile robots. Automatica, 47(1), 2011, 177-184. 